\title{
PROPRIÉTÉS MÉCANIQUES DES VERRES
}

\author{
J. ZARZYCKI
}

\author{
Université de Montpellier 2, Laboratoire des Verres du C. N. R. S., France
}

\begin{abstract}
Résumé. - La résistance mécanique des verres usuels s'écarte très notablement des valeurs théoriquement prévisibles; elle est conditionnée par les phénomènes de fracture fragile et de fatigue statique. Le présent exposé est destiné à servir d'introduction à l'étude de ces aspects des propriétés mécaniques des verres.

Après un bref rappel de la théorie des microfissures (Inglis-Griffith), on aborde l'approche moderne utilisant les méthodes de la dynamique des fractures (Irwin). Le concept de facteur de concentration de contrainte $K_{I}$ permettra de définir le facteur de concentration de contrainte critique $K_{\text {IC }}:$ on examinera sa relation avec l'énergie de surface de fracture (Orowan) caractéristique du matériau. On passera en revue quelques méthodes permettant de mesurer ces quantités et de déterminer la relation fondamentale entre la vitesse de propagation des fractures et $K_{\mathrm{I}}$. Ces méthodes permettent de vérifier la validité de la théorie de corrosion sous contrainte (Charles-Hillig); en combinant ces mesures avec certains tests, il est possible d'effectuer des prévisions sur le comportement mécanique longue durée des verres dans des applications technologiques particulières.
\end{abstract}

\begin{abstract}
The mechanical strength of current glasses falls far below the maximum theoretical values; it is conditionned by brittle fracture and static fatigue. This paper is intended to serve as an introduction to the study of these aspects of the mechanical properties of glasses.

After a brief review of the Inglis-Griffith flaw theory, the modern point of view using Irwin's fracture dynamics is described. Stress-concentration factor $K_{I}$ and the critical $K_{I C}$ are defined and their relationship with surface fracture energy after Orowan is given. Methods of determining these quantities are indicated. The relationship between fracture velocity and $K_{\mathrm{I}}$ shows the validity of the Charles-Hillig stress-corrosion theory. Combining these measurements with statistical tests, it is possible to forecast the long-range mechanical behaviour of glasses in certain technological applications.
\end{abstract}

1. Introduction. - Dans un domaine de températures suffisamment éloigné de la zone de transition, le verre est un solide parfaitement élastique - il peut même être considéré comme un solide élastique idéal - qui obéit à la loi de Hooke sans qu'un comportement plastique puisse être décelé avant la rupture. Celle-ci survient brutalement, sans avertissement préalable à l'endroit où une contrainte d'extension limite a été dépassée. C'est là le comportement type d'un matériau fragile.

Les essais pratiques montrent une dispersion considérable de la charge de rupture pour des échantillons apparemment identiques si bien que pour caractériser la résistance mécanique des matériaux vitreux, on est obligé de recourir à des évaluations statistiques sur un nombre élevé d'échantillons.

On a pu montrer que ce comportement type dépend essentiellement de l'état de surface du verre, qui constitue le point faible où s'initient les fractures.

Placé dans l'atmosphère ambiante le verre voit sa résistance mécanique évoluer - la charge de rupture statique décroît lorsque le temps d'application de la contrainte croît - c'est là la manifestation d'un phénomène désigné sous le nom de fatigue statique.
Ces deux phénomènes de rupture fragile et de fatigue statique dominent le comportement mécanique - et imposent des limites aux applications technologiques du verre. Etant donnée l'importance pratique de ce matériau, de très nombreux travaux ont été éffectués pour essayer de comprendre les mécanismes sousjacents et trouver des remèdes permettant d'améliorer le comportement mécanique du verre - les solutions généralement adoptées consistent en une mise en état de précontrainte des couches superficielles - c'est là le principe de la trempe thermique ou de la trempe chimique du verre.

Le sujet étant très vaste, l'exposé qui suit sera limité au problème de la caractérisation de la résistance à la rupture du verre. Les exemples seront pris parmi les verres d'oxydes, les plus importants au point de vue pratique, mais l'essentiel restera valable pour les solides vitreux en général.

2. Résistance cohésive théorique du verre. $-\mathrm{La}$ résistance cohésive théorique d'un matériau dépend des forces de liaison s'exerçant entre les atomes constitutifs de la structure ; elle peut constituer une mesure valable des contraintes qui doivent être dépassées pour assurer la fracture. Ceci permet en 
principe de comparer entre elles les résistances potentielles de différents matériaux et pour un matériau donné fournit une indication sur la charge de rupture maximale prévisible.

Classiquement [1], on considère la fracture comme le processus de séparation du solide en deux parties, chacune étant limitée par une surface nouvelle dite surface de fracture. Des forces attractives variables pendant le processus de séparation agissent à travers ces interfaces - leur maximum donne la résistance cohésive maxima du matériau. En approximant ce comportement par une fonction potentielle caractéristique du matériau, p. ex. un potentiel de Morse (utilisable dans le cas des liaisons covalentes) :

$$
U=U_{0}\left[\mathrm{e}^{-2 a\left(x-x_{0}\right)}-2 \mathrm{e}^{-a\left(x-x_{0}\right)}\right]
$$

où $U$ représente l'énergie potentielle par unité d'aire des surfaces de fracture séparées par une distance $x$; on en déduit la contrainte $\sigma=-\partial U / \partial x$ sur ces surfaces, qui passe par un maximum $\sigma_{\text {th }}$ pour $\partial^{2} U / \partial x^{2}=0$ (Fig. 1).

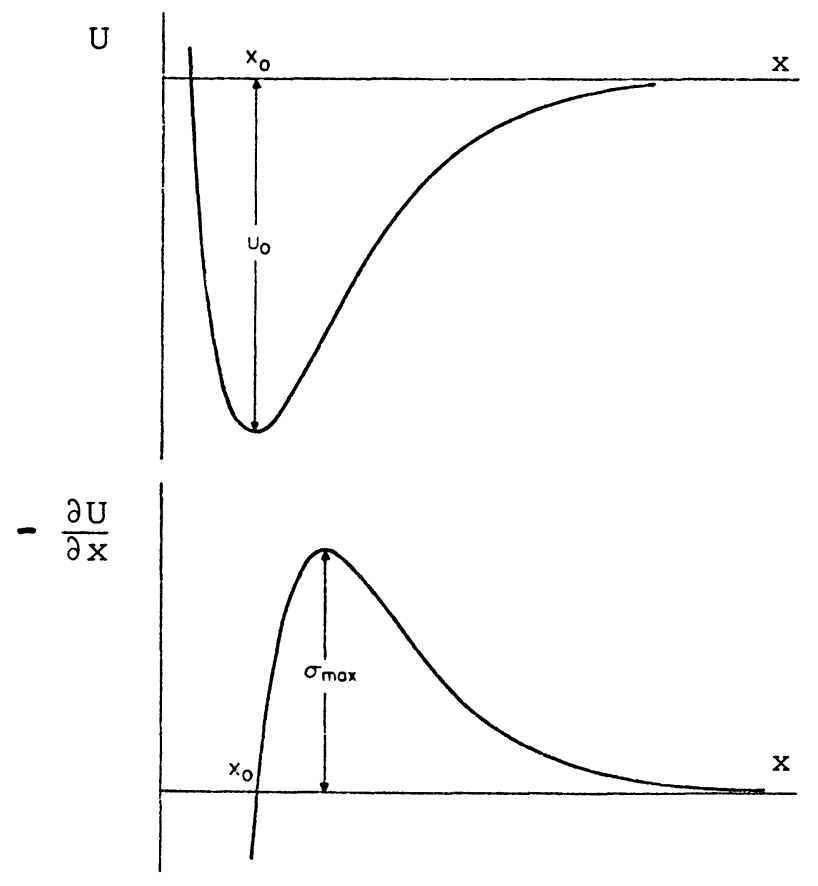

FIG. 1. - Potentiel de liaison $U$ et la force de liaison $-\partial U / \partial x$.

Les constantes $U_{0}, x_{0}$ et $a$ qui entrent dans la définition de la fonction potentielle peuvent être obtenues à partir des propriétés mesurables du matériau. La distance d'équilibre $x_{0}$ en l'absence de la contrainte est déterminée par la distance entre les atomes voisins (ex. Si-O pour $\mathrm{SiO}_{2}$ ) et résulte de la condition

$$
\left(\frac{\partial U}{\partial x}\right)_{x=x_{0}}=0
$$

Le paramètre $U_{0}$ de la fonction potentielle dépend de l'énergie de surface $\gamma$ des surfaces créées au cours de la fracture :

$$
2 \gamma=\int_{x_{0}}^{\infty} \frac{\partial U}{\partial x} \mathrm{~d} x=-U_{0}
$$

et la loi de Hooke permet de relier la courbure de $U$ au module d'Young $E$ du solide :

$$
\mathrm{d} \sigma=E \frac{\mathrm{d} x}{x_{0}}
$$

soit :

$$
E=x_{0}\left(\frac{\partial^{2} U}{\partial x^{2}}\right)_{x=x_{0}}
$$

Dans ces conditions, la contrainte de rupture $\sigma_{\text {th }}$ ou résistance cohésive théorique maxima, correspondant au maximum de $\partial U / \partial x$ s'écrit :

$$
\sigma_{\text {th }}=\sqrt{\frac{\gamma E}{4 x_{0}}} .
$$

Pour le verre de silice $\mathrm{SiO}_{2}$, ce calcul conduit, suivant les estimations de $\gamma$ et $x_{0}$ à des valeurs comprises entre :

$$
1,8 \times 10^{10} \text { et } 2,2 \times 10^{10} \mathrm{Nm}^{-2} .
$$

Ces résistances théoriques sont comparables aux valeurs mesurées expérimentalement dans des circonstances particulières, exceptionnellement favorables, par exemple [1]:

$$
\begin{array}{ll}
1,35 \times 10^{10} \mathrm{Nm}^{-2} & \begin{array}{l}
\text { pour une silice, polie au feu, } \\
\text { mesurée à }-196^{\circ} \mathrm{C}
\end{array}
\end{array}
$$

ou
$1,47 \times 10^{10} \mathrm{Nm}^{-2}$ pour une fibre de silice, mesurée à $-296^{\circ} \mathrm{C}$.

Mais d'une manière générale, les valeurs généralement mesurées pour $\mathrm{SiO}_{2}$ avoisinent plutôt $1 \times 10^{8} \mathrm{Nm}^{-2}$, c'est-à-dire sont environ 100 fois plus faibles que les valeurs théoriques calculées.

Quant aux verres silico-calco-sodiques industriels, leur résistance peut descendre couramment à $3 \times 10^{7} \mathrm{Nm}^{-2}$, c'est-à-dire à des valeurs plus de 1000 fois inférieures aux valeurs maxima prévisibles. De plus, les essais montrent une dispersion considérable que l'on peut relier à l'état de surface de l'échantillon, les spécimens polis au feu et préservés de tout contact mécanique avant les essais montrant une résistance moyenne plus élevée mais aussi une plus grande dispersion dans les résultats (Fig. 2).

Cette différence dans le comportement des différents échantillons et l'écart considérable entre les valeurs théoriquement prévisibles et celles atteintes dans la pratique ont suscité de nombreuses théories. 


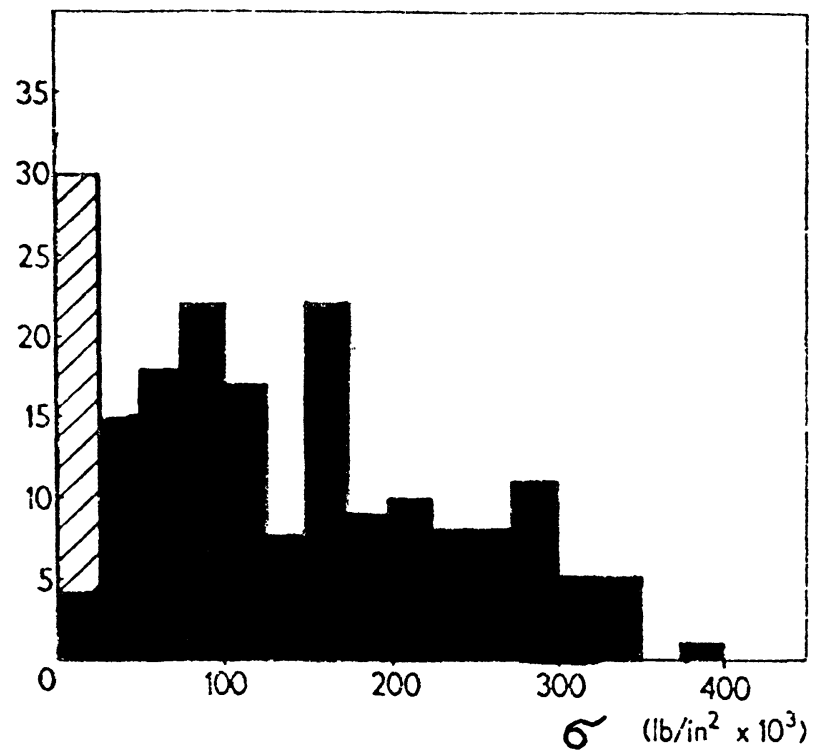

Fig. 2. - Histogrammes de fréquence de la résistance mécanique $\sigma$ d'un verre silico-sodo-calcique; partie hachurée : baguettes ordinaires; partie en noir plein: baguettes vierges. D'après [11].

3. Théorie de microfissures. - Les premières explications des basses valeurs de la résistance mécanique des verres observées dans la pratique ont été données par Griffith [2,3]. Cet auteur a suggéré que tous les échantillons de verre courants contiennent des microfissures ou failles superficielles résultant des manipulations et qui agissent comme concentrateurs des contraintes. L'explication de Griffith est fondée sur les travaux d'Inglis [4] qui a calculé la répartition des contraintes dans une plaque mince comportant un trou elliptique et soumise à une tension uniforme $\sigma_{\mathrm{L}}$ (Fig. 3). Si l'on désigne par $c$ la longueur de demigrand axe de l'ellipse et par $\rho$ le rayon de courbure en

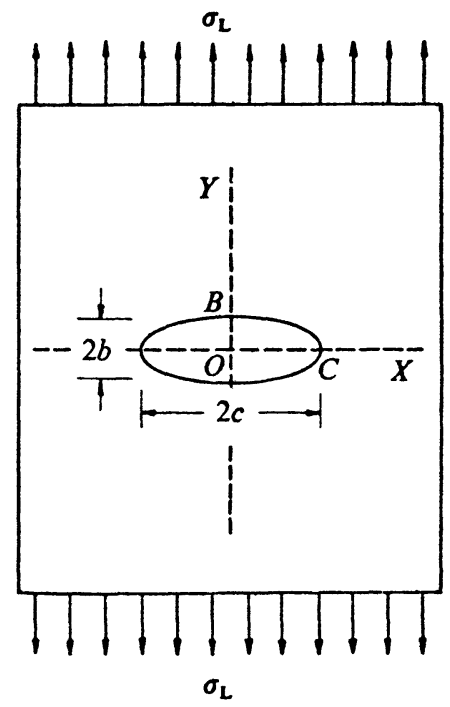

Fig. 3. - Plaque contenant un trou elliptique, soumise à une tension uniforme $\sigma_{\mathrm{L}}$.
$C$, la plus grande contrainte $\sigma_{y y}$ est produite au sommet du grand axe et vaut :

$$
\sigma_{Y Y}=2 \sigma_{\mathrm{L}} \sqrt{\frac{\bar{C}}{\rho}}
$$

Le rapport $2 \sqrt{C / \rho}$ est le facteur de concentration de contrainte. En supposant qu'au moment de la rupture $\sigma_{y y}=\sigma_{\mathrm{th}}$, la contrainte de rupture $\sigma_{\mathrm{f}}$ est :

$$
\sigma_{\mathrm{f}}=\sigma_{\mathrm{th}} / 2 \sqrt{\frac{\bar{C}}{\rho}}
$$

Or le facteur de concentration de contrainte qui dépend surtout de la forme des fissures peut prendre des valeurs notablement supérieures à 1 pour des fissures étroites. A la limite, pour une fissure approchant les dimensions de l'ordre des distances interatomiques à son extrémité, si p. ex. :

$\rho=2 \AA \quad$ et $\quad c=1 \mu, \quad 2 \sqrt{\frac{C}{\rho}}=2 \sqrt{\frac{10^{4}}{2}} \sim 140 ;$

ce qui permet de rendre compte de la baisse des valeurs observées dans la pratique par rapport aux résistances théoriques.

Malgré ce pas fondamental franchi, l'explication du mécanisme de fracture demeurait obscure. En particulier, si l'analyse d'Inglis demeurait valable jusqu'aux dimensions atomiques, pourquoi dans la pratique des fissures plus grandes se propagent-elles plus facilement que les petites ? Et quelle est la signification physique du rayon de courbure d'une fissure réelle ?

Griffith reprit alors la question en proposant un modèle en termes d'un processus thermodynamique réversible.

Il a considéré le bilan d'énergie potentielle dans une plaque mince infinie d'épaisseur $t$ soumise à une contrainte d'extension $\sigma$ et dans laquelle on a introduit une faille elliptique de demi-grand axe $c$ (Fig. 3).

$U_{\text {I }}$ étant l'énergie potentielle de la plaque tendue avant l'introduction de la fissure, le calcul donne l'énergie potentielle :

$$
U=U_{\mathrm{I}}-\frac{\Pi \sigma^{2} c^{2} t}{E}+4 c t \gamma
$$

où $E$ est le module d'Young et $\gamma$ l'énergie de surface solide.

$\mathrm{Au}$ cours d'une extension $\mathrm{d} c$ de la fissure, le terme d'énergie mécanique décroît et le terme d'énergie superficielle croît ; à l'équilibre :

$$
\frac{\mathrm{d} U}{\mathrm{~d} c}=0
$$

ce qui donne :

$$
\frac{\Pi \sigma^{2} c}{E}=2 \gamma
$$


Comme $\partial^{2} U / \partial c^{2}<0$, l'équilibre est instable, et l'équation (7) définit ainsi la contrainte amenant l'instabilité de la fissure :

$$
\sigma_{\mathrm{f}}=\sqrt{\frac{2 e \gamma}{\Pi c}} .
$$

Dans le cas d'une déformation plane (plaque épaisse), le même raisonnement conduit à :

$$
\sigma_{\mathrm{f}}=\sqrt{\frac{2 E\left(1-v^{2}\right) \gamma}{\Pi c}}
$$

$v$ étant le nombre de Poisson du matériau. Pour vérifier sa théorie, Griffith a procédé à une série d'expériences en introduisant systématiquement des fissures macroscopiques de longueur connue $2 c$ dans les parois des ampoules en verre dont il mesurait la résistance à l'éclatement par augmentation de la pression intérieure; il a pu ainsi vérifier la forme générale de sa relation :

$$
\sigma_{\mathrm{f}} \sqrt{c}=\mathrm{Cte}_{\mathrm{te}}=2,63 \times 10^{5} \mathrm{Nm}^{-3 / 2} .
$$

En combinant cette relation avec la définition (4) et en prenant $\rho \sim 5 \times 10^{-10} \mathrm{~m}$, ceci conduit à une résistance de cohésion théorique du verre

$$
\sigma_{\text {th }} \sim 2,3 \times 10^{10} \mathrm{Nm}^{-2} .
$$

Cependant, compte tenu de la valeur du module d'Young, la déformation correspondante serait de 0,3 à 0,4 et il est difficile d'admettre que la loi de Hooke serait encore valable, la relation force de liaison-séparation devenant certainement non linéaire avant la rupture.

Avec son hypothèse de failles relative à l'initiation des fractures et son concept d'équilibre énergétique déterminant la propagation, Griffith a établi des bases solides d'une théorie générale de la fracture du verre et des solides fragiles en général.

De nombreuses discussions ont été consacrées aux mérites respectifs des deux critères proposés par Griffith. En faisant un raisonnement tenant également compte des échanges calorifiques, Dorémus [5] a pu établir à partir des deux principes de thermodynamique que le bilan énergétique de Griffith constitue une condition nécessaire mais non suffisante de propagation et d'autre part que l'existence des rayons de courbure inférieurs à une dizaine de distances interatomiques est incompatible, le système agrandissant automatiquement $\rho$ par fluctuation jusqu'à une valeur thermodynamiquement acceptable.

L'hypothèse de Griffith explique la dispersion des essais de caractérisation mécanique des verres, l'introduction des failles étant à la fois aléatoire et cumulative, des contacts mécaniques et des agressions chimiques fournissant continuellement de nouvelles failles superficielles.

De très nombreuses théories statistiques des failles ont été développées ; elles ont pour but la prédiction de la résistance théorique, en admettant des distri- butions diverses des longueurs des failles, pour un lot d'échantillons donnés. Elles connaissent des limitations sévères car les failles les plus vulnérables seront fatalement situées dans les queues de toutes les fonctions de distribution statistiques - et échappent donc facilement à la prédiction.

Le problème en est resté là jusqu'aux années soixante, où l'on assiste à un renouveau grâce notamment aux travaux d'Irwin [6] sur la fracture fragile, motivés par la demande générale des critères de résistance mécanique plus sûrs dans les réalisations technologiques avancées de l'après-guerre.

4. Aspect moderne. Dynamique des fractures. Les travaux de cet auteur ont été orientés dans deux directions distinctes qui constituent des généralisations des deux critères de Griffith.

4. 1 Point de Vue d'inTensité de la CONTRAinte. En reprenant le premier concept on peut dire que la fragilité du verre est due au fait que des failles tendent à redistribuer les champs de contraintes internes en provoquant des concentrations élevées de contraintes aux sommets des fissures. La fracture survient lorsque ces champs atteignent des valeurs critiques. Pour déterminer la résistance à la fracture, il faut donc étudier la distribution des contraintes autour des fissures. Irwin [6] et Williams [7] ont déterminé le champ des contraintes le plus général au voisinage $\mathrm{du}$ sommet d'une fissure infiniment mince dans un matériau continu supposé infini.

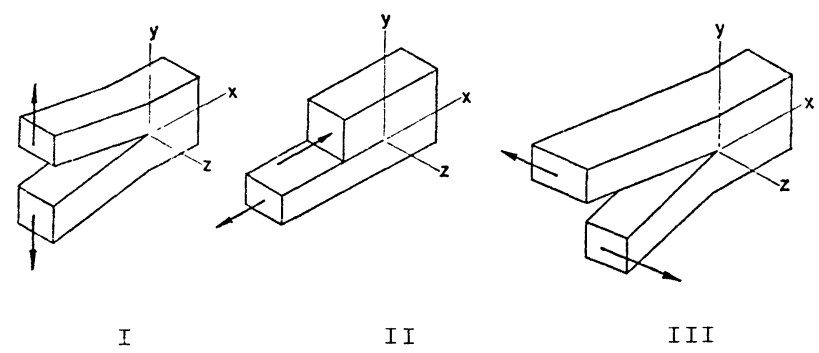

Fig. 4. - Modes principaux de mise en charge d'une fracture. D'après [9].

Trois modes principaux de mise en charge peuvent être distingués (Fig. 4), le cas le plus général s'obtenant par une superposition linéaire de ces trois modes fondamentaux. Le mode I, dit mode ouvrant, est le plus adapté au cas du verre, les modes II (par glissement) et III (par cisaillement) étant moins importants car le verre cède généralement à la traction.

Dans un système de coordonnées cylindriques $(r, \theta)$ rapportés à l'extrémité $o z$ de la fissure (Fig. 5), on peut montrer que les composantes $\sigma_{i j}$ du tenseur de contrainte sont de la forme :

$$
\sigma_{i j}=-\frac{K}{\sqrt{2 \Pi r}} f_{i j}(\theta)
$$




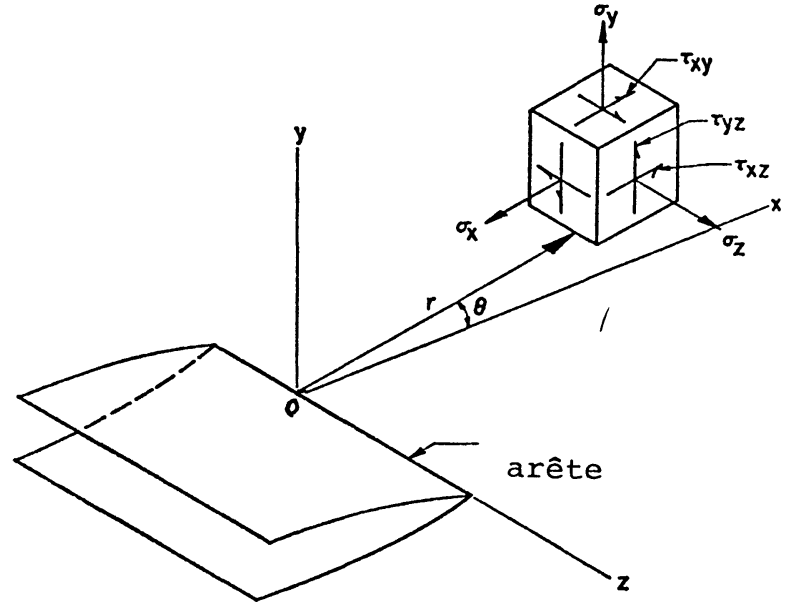

Fig. 5. - Coordonnées définissant le champ de contraintes au voisinage de l'arête de la fissure. D'après [9].

$\sigma_{i j}$ apparait ainsi comme un produit de trois facteurs distincts. La dépendance radiale est toujours proportionnelle à $r^{-1 / 2}$, quel que soit le mode de charge, géométrie de la fissure ou la valeur de la charge. La variation angulaire donnée par $f_{i j}(\theta)$ ne dépend que du type de la contrainte et du mode d'application des charges, mais pas de la valeur de la charge ellemême, ni de la géométrie de la fissure. Ces fonctions déterminent donc la distribution du champ local. Le facteur $K$ dit facteur de concentration de contrainte ne dépend que de la géométrie de la fissure et de la manière dont sont appliquées les charges extérieures. $K$ déterminera donc l'intensité du champ local. Le résultat le plus significatif de cette analyse est donc la séparation des contraintes (et des déplacements) en des portions radiale, angulaire et celle dépendante de l'intensité de la contrainte.

Les distributions radiale et angulaire seront identiques pour un mode donné tandis que le niveau des contraintes ne dépendra que de $K$. Pour le mode I, le facteur de l'intensité de contrainte $K_{\mathrm{I}}$ sera de la forme : $K_{\mathrm{I}}=\alpha S$ où $S$ est la charge appliquée et $\alpha$ un facteur géométrique calculable pour une configuration donnée. Les figures 6 à 8 donnent des exemples importants de géométries utilisées dans les essais et pour lesquelles le calcul de $K_{\mathrm{I}}$ est indiqué. De

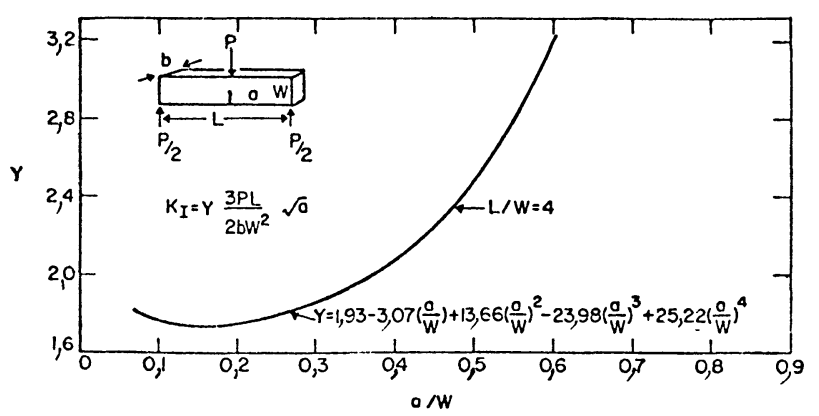

Fig. 6. - Poutre entaillée fléchie en trois points. Calcul de $K_{I}$. D'après [8].

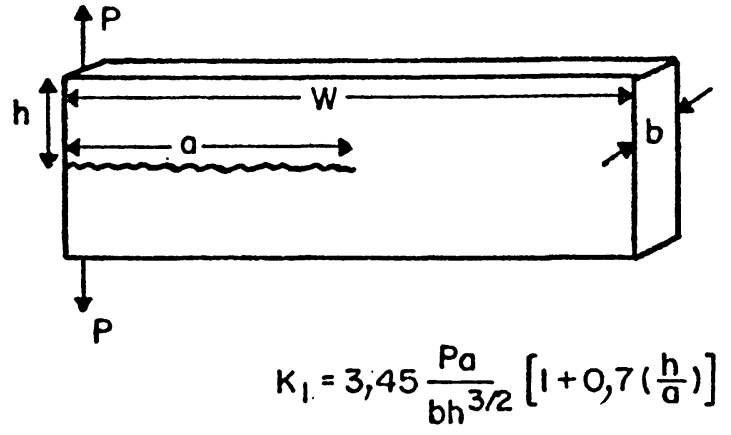

Fig. 7. - Cantilever double. Calcul de $K_{\mathrm{I}}$. D'après [8].

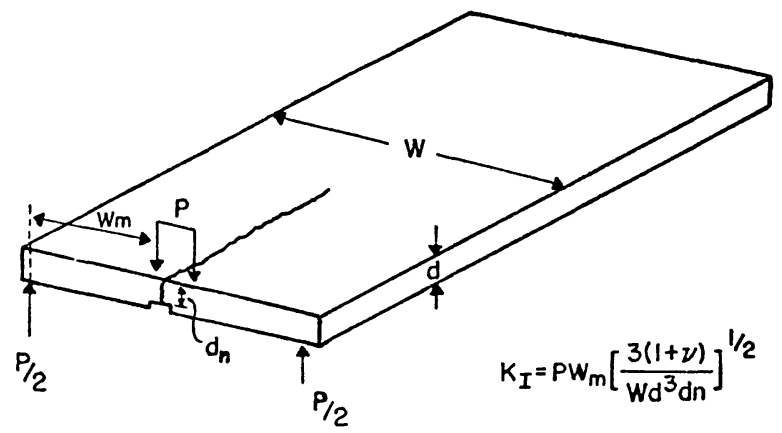

Fig. 8. - Plaque mince en torsion double. Calcul de $K_{\mathbf{I}}$. D'après [8]

nombreuses autres configurations ont été utilisées $[8,9]$.

D'après les formules (10), les $\sigma_{i j}$ deviennent infinis à la pointe de la fissure pour $\sigma=0$. Ceci ne correspond évidemment pas à la réalité, mais constitue une singularité mathématique due aux hypothèses du matériau continu et d'une fissure infiniment mince et qui disparaît pour un matériau réel à structure atomique discrète. Dans un matériau réel la perturbation au voisinage immédiat de la pointe de la fissure, liée au caractère discret de la matière n'aura toutefois qu'une influence négligeable sur le champ de contraintes plus éloigné (Principe de St Venant).

Le fait essentiel de cette analyse est la correspondance biunivoque entre la contrainte maximale d'extension au voisinage de la fissure et la valeur du facteur d'intensité de contrainte. A la fracture, on pourra donc caractériser le matériau par une valeur $K_{\mathrm{c}}$ critique de $K$, p. ex. $K_{\mathrm{IC}}$ pour le mode I.

$K_{\text {IC }}$ sera donc une constante intrinsèque du matériau, dépendant des processus physiques se produisant à la pointe de la fissure lors de la fracture, mais indépendante de la géométrie, forme de l'échantillon ou du type d'essai. $K_{\text {IC }}$ permet de chiffrer la difficulté de propager une fissure dans le matériau, mais est indépendant de la distribution des failles etc. qui constituent les difficultés des caractérisations primitives.

4.2 PoINT DE VUE DE L'ÉNERGIE DE SURFACE. Cette deuxième approche a pour but d'incorporer les 
solutions précédentes données par la théorie de l'élasticité dans le critère d'équilibre énergétique de Griffith.

On démontre tout d'abord que l'énergie mécanique libérée au cours d'une augmentation de la fissure est indépendante de la configuration des charges.

On définit dès lors une force généralisée d'extension de la fissure, à déplacement $u$ constant :

$$
G=-\left(\frac{\partial U_{\mathrm{E}}}{\partial c}\right)_{u}
$$

qui représente le taux de la libération de l'énergie élastique $U_{\mathrm{E}}$ par accroissement de la longueur $c$ de la fissure. Une relation générale existe entre le paramètre $G$ et le facteur de concentration de contrainte $K$.

Par intégration des équations du champ de contrainte et déplacements, Irwin a pu montrer que dans le cas du mode I cette relation s'écrit :

$$
G=\frac{K_{\mathrm{I}}^{2}}{E}
$$

pour le cas d'un système de contraintes plan, et

$$
G=\frac{K_{I}^{2}\left(1-v^{2}\right)}{E}
$$

pour le cas d'une déformation plane. On montre de plus que les $G$ pour les différents modes sont additifs.

Le concept de Griffith s'introduit dès lors en égalant à l'équilibre les forces $G$ au double de l'énergie de surface $\gamma$ ( 2 surfaces créées par la rupture) :

$$
G_{\mathrm{c}}=\frac{K_{\mathrm{IC}}^{2}}{E}=2 \gamma
$$

pour la contrainte plane, ou :

$$
G_{\mathrm{c}}=\frac{K_{\mathrm{IC}}^{2}\left(1-v^{2}\right)}{E}=2 \gamma
$$

pour la déformation plane.

Cette formulation a été généralisée par Orowan [10] au cas des matériaux pouvant comporter une zone non linéaire au voisinage de la pointe de la fissure, où peuvent apparaître des phénomènes dissipatifs. Ceci conduit à remplacer $\gamma$ par un terme $\Gamma$ appelé énergie de surface de fracture ou travail de fracture qui se réduit à $\gamma$ pour un solide fragile idéal.

L'extension Irwin-Orowan du concept de Griffith s'écrit dès lors :

$$
G_{\mathrm{c}}=2 \Gamma \text {. }
$$

Cette formulation nécessite cependant un modèle détaillé de la pointe de fissure pour chiffrer les termes dissipatifs de volume - elle perd donc en puissance d'analyse par rapport à la formulation idéale où l'énergie de surface est déterminée d'une manière unique par l'aire de la surface de fracture créée.
5. Méthodes de mesure. - Il y a trois méthodes expérimentales pour déterminer les paramètres critiques de fracture $K_{\mathrm{IC}}$ ou $G_{\mathrm{IC}}$, liés entre eux par les relations (12) ou (13) $[1,8]$.

5.1 LA MÉTHOdE ANALYTIQUe. - Elle consiste à calculer $K_{\mathrm{I}}$ en fonction des charges appliquées, longueur de la fissure et géométrie de l'échantillon. $K_{\text {IC }}$ est obtenu en mesurant la charge amenant la fracture du système. Pour des conditions physiques identiques, $K_{\mathrm{IC}}$ doit se montrer indépendant de la méthode d'essai utilisé.

Les géométries pratiques utilisées les plus usuelles comprennent un essai de flexion sur une barrette entaillée (Fig. 6), ou le double cantilever (Fig. 7).

5.2 LA MÉTHODE DE COMPLAISANCE. - Elle repose sur l'emploi de l'équation :

$$
G=\frac{1}{2} F^{2}\left(\frac{\partial \lambda}{\partial c}\right)_{F}
$$

fournie par la théorie générale de l'élasticité et qui lie la complaisance $\lambda=u / F$ du système subissant un déplacement $u$ du point de charge sous une charge $F$ constante. La mesure s'effectue sur une série d'échantillons comportant des fissures de longueur variable $c$.

5.3 LA MÉTHODE DE TRAVAIL DES FORCES DE FRACTURE. - Elle consiste à déterminer le travail pour propager une fracture sur une section complète d'une éprouvette entaillée dans un essai de flexion sur trois points. La méthode repose sur le fait que lorsque la fracture se propage, la complaisance $\lambda$ diminue et la propagation devient continue et stable pour une charge décroissante (Fig. 9). A partir de l'aire sous-tendue par la courbe contrainte-déplacement enregistrée qui représente le travail total effectué on obtient $G_{\text {IC }}$ en divisant ce travail par la demisurface totale de fracture. Le tableau I contient des

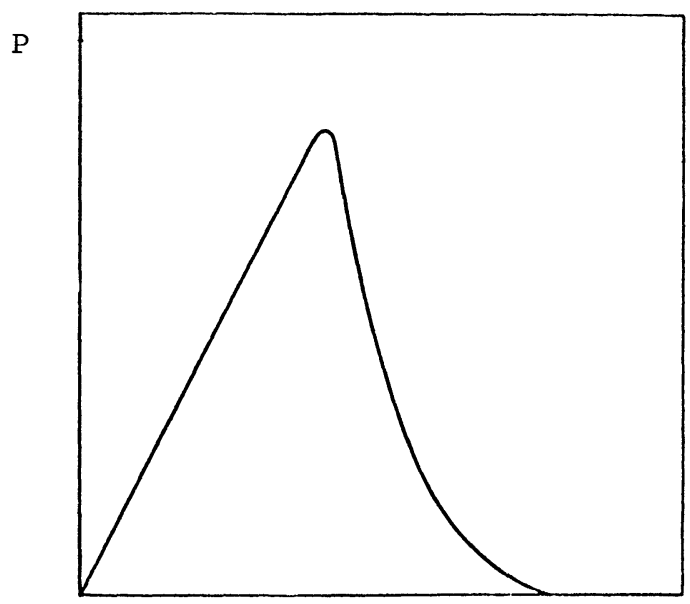

$\mathrm{u}$

FIG. 9. - Courbes charge P-déplacement u dans une expérience de détermination du travail de fracture. D'après [8] 


\section{TABLEAU I}

Energie de surface de fracture $\gamma$ des verres obtenus par la méthode de double cantilever [1]

Silice vitreuse $\gamma\left(\mathrm{Jm}^{-2}\right)$

Verre silico-sodo-calcique

4,32

Borosilicate

4,75

4,65

Aluminosilicate

3,50

valeurs de $\gamma$ obtenues par la méthode du double cantilever. L'introduction de $\gamma$ dans la formule (9) conduit à des valeurs de résistance cohésive de l'ordre de $2 \times 10^{10} \mathrm{Nm}^{-2}$.

6. Effets d'environnement. Fatigue statique. Nous avons supposé jusqu'à présent que la fracture est simplement contrôlée par la géométrie des failles et des propriétés intrinsèques du matériau. En fait, l'environnement exerce une action de réduction de la résistance comme le montrent des essais classiques dits de fatigue statique (Fig. 10).

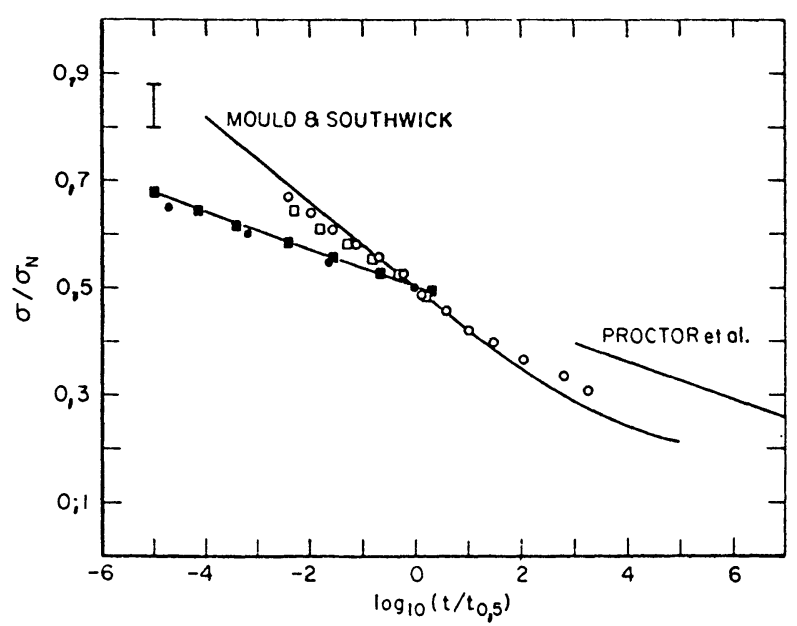

Fig. 10. - Fatigue statique des verres. Comparaison des résultats obtenus à partir de la propagation des fractures avec la courbe de fatigue universelle. D'après [19].

La résistance du verre dépend de la vitesse de charge; le verre chargé rapidement apparaît plus résistant et on observe un temps de fracture différée qui s'accroît lorsque la charge diminue. Ces effets n'apparaissent qu'en présence de la vapeur d'eau et ont été expliqués par Charles et Hillig [14] par un phénomène de corrosion sous contrainte - la réaction d'hydrolyse progressant plus rapidement aux extrémités des fissures où les contraintes sont élevées. Les fissures s'étendent sous contraintes sub-critiques sous l'influence de la réaction chimique :

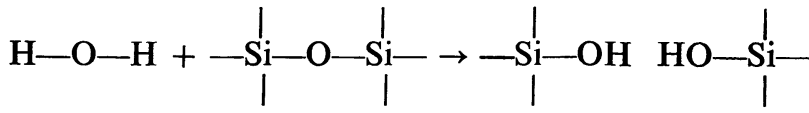

qui provoque des ruptures des liaisons dans la fissure (Fig. 11) jusqu'à ce que le critère de Griffith soit satisfait au point où la fracture caractéristique se produit.

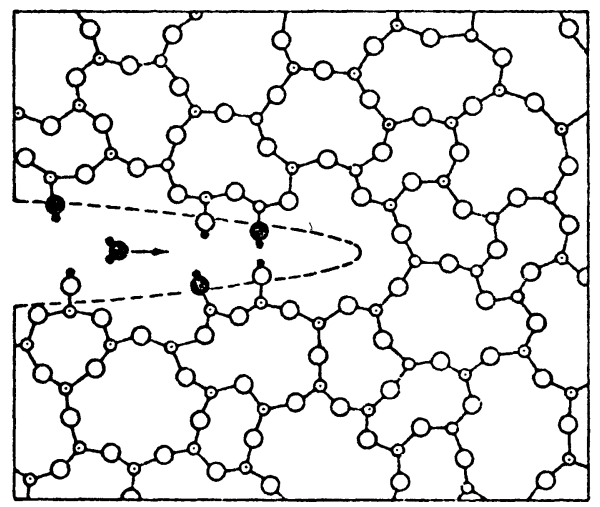

FIG. 11. - Modèle bidimensionnel d'une fissure induite par l'eau dans le verre de silice. Les atomes en plein représentent des espèces empruntées à l'environnement $\left(\mathrm{H}_{2} \mathrm{O}\right)$. D'après [15].

Les expériences de Wiederhorn [16] sur la propagation des fissures sub-critiques dans différents environnements sont résumées dans la figure 12, qui montre la relation entre la vitesse de propagation $v$ et le facteur de concentration de contrainte $K_{\mathrm{I}}$.

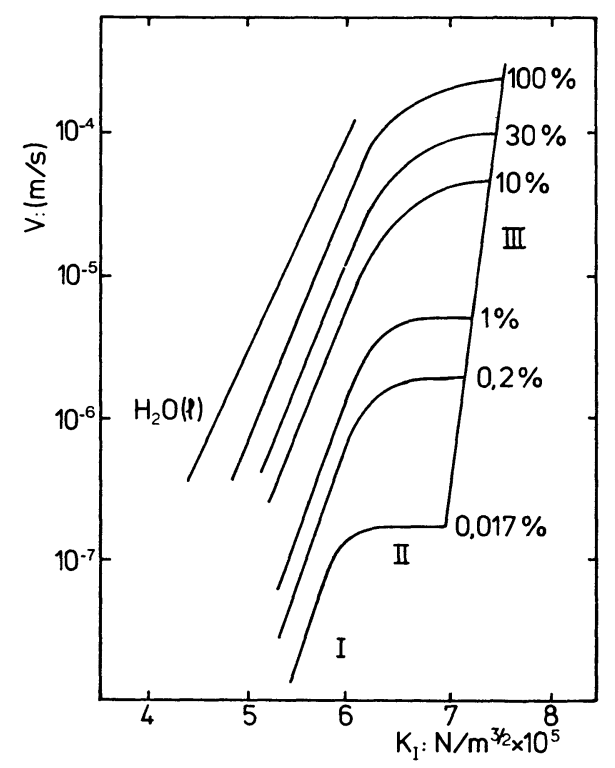

FIG. 12. - Dépendance de la vitesse de fracture $v$ du facteur $K_{I}$ pour le verre $\mathrm{SiO}_{2}-\mathrm{Na}_{2} \mathrm{O}-\mathrm{CaO}$. D'après [16].

Pour chaque concentration en vapeur d'eau (indiquée en $\%$ sur la figure), la courbe présente trois régions distinctes. Pour des valeurs de

$$
K_{\mathrm{I}}<6 \times 10^{5} \mathrm{Nm}^{-3 / 2}
$$


environ (région I), la vitesse dépend exponentiellement de $K_{\mathrm{I}}$ et du $\%$ en vapeur d'eau. Dans la région II (plateau) la vitesse est indépendante de $K_{\mathrm{I}}$ mais dépend de l'ambiance; dans la région III enfin la vitesse devient de nouveau dépendante de $K_{\mathrm{I}}$ mais ne dépend plus de l'environnement.

Les régions I et II peuvent être expliquées par la théorie de Charles et Hillig; dans la région I, la vitesse est limitée par la réaction chimique alors que dans la région II, la vitesse est limitée par le transport de vapeur d'eau de l'environnement vers la pointe de la fissure. La région III n'a pas reçu encore d'explication satisfaisante.

L'étude en fonction de la température montre une relation du type :

$$
v=v_{0} \exp \left(-E^{*}+b K_{1}\right) / R T
$$

en accord avec les théories Charles-Hillig.

L'importance de ces mesures réside dans la possibilité d'appliquer ces résultats au problème de la prédiction et de la durée de vie des composants en verre en service [17]. Le temps nécessaire à la rupture peut être évalué à l'aide des concepts de dynamique de fracture.

Le facteur d'intensité de contrainte $K_{\mathrm{I}}$ pour une faille de dimension a et une contrainte appliquée $\sigma_{\mathrm{a}}$ sera de la forme :

$$
K_{\mathrm{I}}=\sigma_{\mathrm{a}} Y \sqrt{a}
$$

où $Y$ est un facteur géométrique (pour les fissures de surface $Y \sim \sqrt{\Pi})$. En dérivant $K_{\mathrm{I}}$ par rapport au temps, on forme l'équation donnant la vitesse de propagation $v=\mathrm{d} a / \mathrm{d} t$ de la fissure.

$$
\mathrm{d} K_{\mathrm{I}} / \mathrm{d} t=\left(\sigma_{\mathrm{a}}^{2} Y^{2} / 2 K_{\mathrm{I}}\right) v
$$

d'où par intégration le temps pour la fracture :

$$
t=\frac{2}{\sigma_{\mathrm{a}}^{2} Y^{2}} \int_{K_{\mathrm{I} i}}^{K_{\mathrm{IC}}} \frac{K_{\mathrm{I}}}{v} \mathrm{~d} K_{\mathrm{I}} .
$$

En connaissant la relation entre $v$ et $K_{\mathrm{I}}$ ainsi que $K_{\mathrm{IC}}$ obtenus expérimentalement, il est possible de déterminer $t$ si l'on connaît le facteur de concentration de contrainte initial $K_{\mathrm{I} i}$.

Pour ceci, deux méthodes peuvent être utilisées [17] :

1. On utilise un mode d'évaluation statistique ; p. ex. en calculant les paramètres de distribution de Weibull [18].

Le résultat conduit à un abaque donnant la probabilité de fracture au bout d'un temps donné sous un niveau de contrainte donné.

2. On procède par essai systématique sur tous les échantillons dans les conditions identiques à celles auxquelles ils seront soumis en service, mais à un niveau supérieur : essai de purge ou proof testing.

Dans ces conditions, il est permis de déterminer le niveau de surcharge de cet essai pour avoir une probabilité de rupture réelle en service pour un temps déterminé.

De tels essais ont été utilisés en particulier pour tester les hublots de cabines spatiales (Skylab) où une garantie totale était recherchée [17].

7. Conclusion. - Les phénomènes de fracture fragile qui limitent sévèrement les applications du matériau verre commencent à être connus en profondeur grâce aux récents développements des méthodes de la dynamique des fractures. Ces méthodes permettent de caractériser les propriétés mécaniques intrinsèques des verres. Les effets dus à la fatigue statique peuvent être chiffrés et fournissent des prévisions qui permettent d'envisager l'extension prochaine des applications techniques de ces matériaux aux domaines jusque-là interdits.

\section{Bibliographie}

[1] Wiederhorn, S. M., dans Mechanical and Thermal Properties of Ceramics, J. B. Wachtman Ed. (Bur. Stand. Special Publ.) 303, p. 217, 1969.

[2] Griffith, A. A., Phil. Trans. Roy. Soc. (London) 221A (1920) 163

[3] Griffith, A. A., Proc. First Intern. Congr. Appl. Mechanics, Delft, p. 55, 1924.

[4] Inglis, C. E., Trans. Inst. Naval Architects (London), 55 (1913) 219.

[5] Doremus, R. H., J. Appl. Phys. 47 (1976) 1833.

[6] Irwin, G. R., J. Appl. Mech. 24 (1957) 361.

[7] Williams, M. L., J. Appl. Mech. 24 (1957) 109.

[8] Evans, A. G. dans Fracture Mechanics of Ceramics, Vol. I, Ed. Bradt (Hasselmann, Lange ; Plenum, New York), p. 17, 1974.

[9] PARIS, P. C., SiH, G. C., dans Fracture Toughness Testing and its Applications (ASTM Spec. Tech. Publ.) 1965381 p. 30.
[10] Orowan, E., Weld. Res. Supp. 34 (1955) 157.

[11] Proctor, B. A., Appl. Mat. Res. 3 (1964) 28.

[12] Wiederhorn, S. M., J. Non Cryst. Solids 19 (1975) 169.

[13] Mould, R. E., dans « Fundamental Phenomena in Materials Science » (Plenum, N. Y.) Vol. 4, p. 119 (1967).

[14] Charles, R. J., Hillig, W. B. dans «Symp. Mechanical Strength and Ways of Improving it " (Florence, USCV) (1962) p. 25.

[15] LaWN, B. R., Wilshaw, T. R., Fracture of Brittle Solids Cambridge (1975) p. 165.

[16] Wiederhorn, S. M., J. Am. Cer. Soc. 50 (1967) 407.

[17] Wiederhorn, S. M., Reliability, Life Prediction and Proof Testing of Ceramics, NBSIR Rep. 74-486 (1974).

[18] Weibull, W., A Statistical Theroy of the Strength of Materials-Ingeniör Vetenskaps Akademien Handlingar Nr 151 (1939). 